\title{
Disk Chemistry and Cometary Composition
}

\author{
A. J. Markwick \& S. B. Charnley \\ Space Science Division, NASA Ames Research Center, Moffett Field, \\ CA 94035
}

\begin{abstract}
We describe a theoretical study of protoplanetary disk chemistry. By considering physical conditions similar to that of the protosolar nebula, we attempt to assess the contribution made by material from the cooler nebular regions to cometesimal composition. Calculations are presented which determine the spatial and temporal chemistry of the gas and dust within the 5-40 AU cometforming region of the nebula. We show that there is little radial variation in the solid-state distribution of some molecules which could potentially be parents of the carbon-chain species observed in comets. We conclude that the apparent variation in abundance of $\mathrm{C}_{2}$ and $\mathrm{C}_{3}$ between long- and short-period comets is the result of chemical processing during the $r$ lifetimes and not differences in composition at the time of formation.
\end{abstract}

\section{Introduction}

Short-period comets formed in the nebula roughly where they reside today ( $\sim 30-1000 \mathrm{AU})$ and comprise the Kuiper Belt. On the other hand, long-period comets formed closer to the protosun $(\sim 5-40 \mathrm{AU})$, but dynamical effects led them to be ejected from there into the Oort Cloud (radii $\sim 10^{3}-10^{5} \mathrm{AU}$ ). There is an apparent variation in the coma abundances of $\mathrm{C}_{2}$ and $\mathrm{C}_{3}$ between each comet population, with the short-period comets being more depleted in these molecules (A'Hearn et al. 1995). As $\mathrm{C}_{2}$ and $\mathrm{C}_{3}$ are coma photoproducts (Helbert et al. 2000), any differentiation between the comet families must be due to chemical differences in the nuclear ices of the two populations. Possible nuclear parents are $\mathrm{C}_{2} \mathrm{H}_{2}$ (acetylene) and $\mathrm{CH}_{3} \mathrm{CCH}$ (methyl acetylene). The origin of this differentiation could lie either in the protostellar disk chemistry which occurred during their formation (nature), or in the chemical-processing histories (photolysis, radiolysis, heating) of each family (nurture). Using a model of the chemistry in the protostellar disk, we might hope to discriminate between these two possibilities.

\section{The Model}

The model we have developed is constructed in two stages. First, the physical structure (density, temperature) of the disk is computed. This calculation includes the combined heating effects of viscous dissipation and irradiation by the central object, as well as a 2D treatment of the radiative transfer (Nomura 2002). The structure $(0<r<100 \mathrm{AU})$ of a steady $\alpha$-disk is produced for a central solar-mass object with mass accretion rate $10^{-7}$ solar masses per year. 
Figure 1 shows the associated temperature and density distributions. These results are then used as input for a comprehensive chemical model, described in Markwick et al. (2002). Briefly, the reaction network comprises around 250 species and 2500 reactions of various types including photoprocesses and gasgrain interaction. The latter allows molecules to freeze out onto dust particles and be thermally evaporated as conditions allow. Note that this model does not include any reactions between species on the grain surfaces, except the formation of molecular hydrogen and some ion-grain recombination reactions. This is because there are conceptual problems in attempting to treat surface reactions in such a deterministic model. The model integrates the chemical rate equations at each point for which physical structure is available, and so 2D gas-phase molecular distributions can be produced (see Millar et al. 2003). In this work, we consider only the mid-plane abundances of solid-phase species, which we assume will be incorporated directly into cometary ices as they form.

\section{Results and Discussion}

Figure 2 shows the variation of some solid-phase carbonaceous species in the midplane of the disk. The region between 10 and $40 \mathrm{AU}$ is a significant fraction of the comet-forming region and, encouragingly, we see that, due to the temperature structure of the mid-plane, molecules in the ice are abundant there. If we are to adopt the 'nature' perspective described above, then we might expect to see a difference in the carbon-chain abundances between 10 and $40 \mathrm{AU}$. However, Figure 2 shows that there is no variation in the abundances of these species in the region of interest. The same result is true for all species with 2 and 3 carbon atoms in the model: there is no variation between 10 and $40 \mathrm{AU}$. The reason for this is quite straightforward. The gas-grain interaction in the model is described by 'freeze-out'. This is a balance between sticking collisions between molecules and grains, and the return of these molecules by evaporation from grain surfaces. Thermal desorption depends on the nebular dust (and gas) temperature, $T$, and on the binding energy of the molecule to the dust grain, $E_{b}$, a material property. The temperature structure in the mid plane is such that the thermal desorption rate $\left(\propto \exp \left[-E_{b} / k T\right]\right)$ effectively switches the solid-state abundances between negligible and significant: binding energies are simply not low enough to allow a variation across the comet-forming region, where the temperature varies by only about $10 \mathrm{~K}$. Hence, using the model described here, we conclude that any differences in carbon-chain composition between long- and short-period comets arise due to their different processing histories (see Stern 2003).

This work was supported by NASA's Origins of Solar Systems Program with funds allocated by NASA Ames under Interchange No. NCC2-1412. AJM is a National Research Council postdoctoral research associate.

\section{References}

A'Hearn M. et al., 1995, Icarus 118, 223

Helbert, J., Rauer, H., Boice, D., Huebner, W. F., 2000, BAAS, 32, 1071

Markwick A. J., Ilgner M., Millar T. J., Henning Th., 2002, A\&A, 385, 632

Millar T. J., Nomura H., Markwick A. J., Ap\&SS, 285, 761 


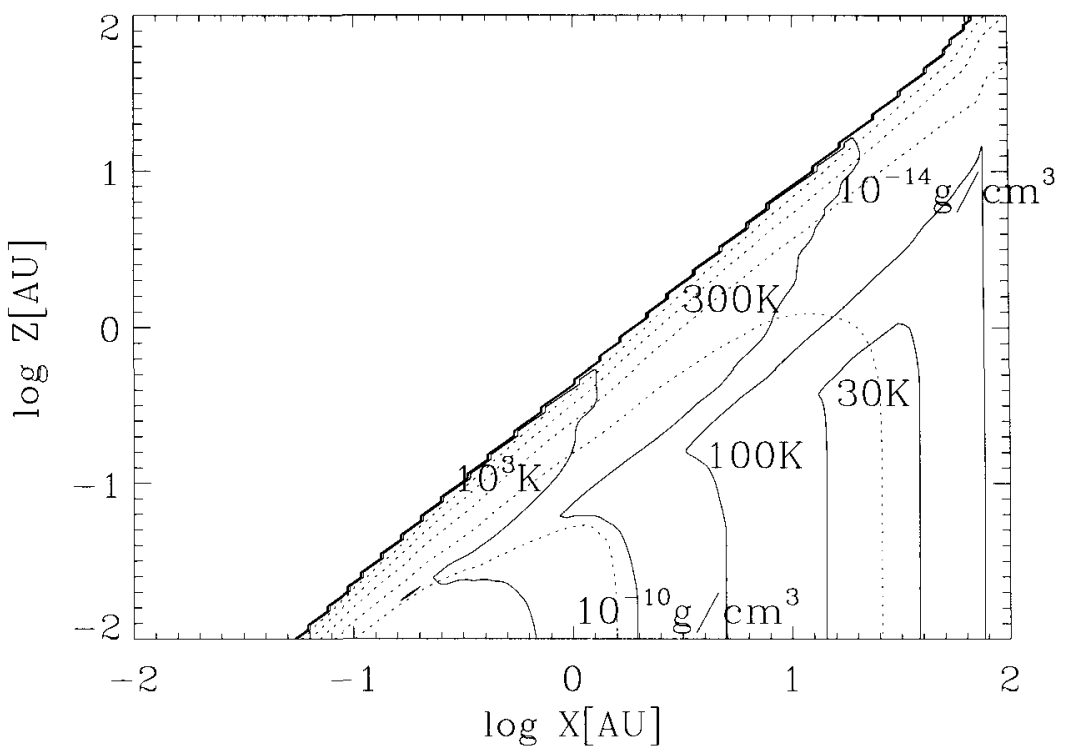

Figure 1. The two-dimensional structure of the disk for $r<100$ A.U.

Nomura H., 2002, ApJ, 567, 587

Stern, S. A. 2003, Nature 424, 639 


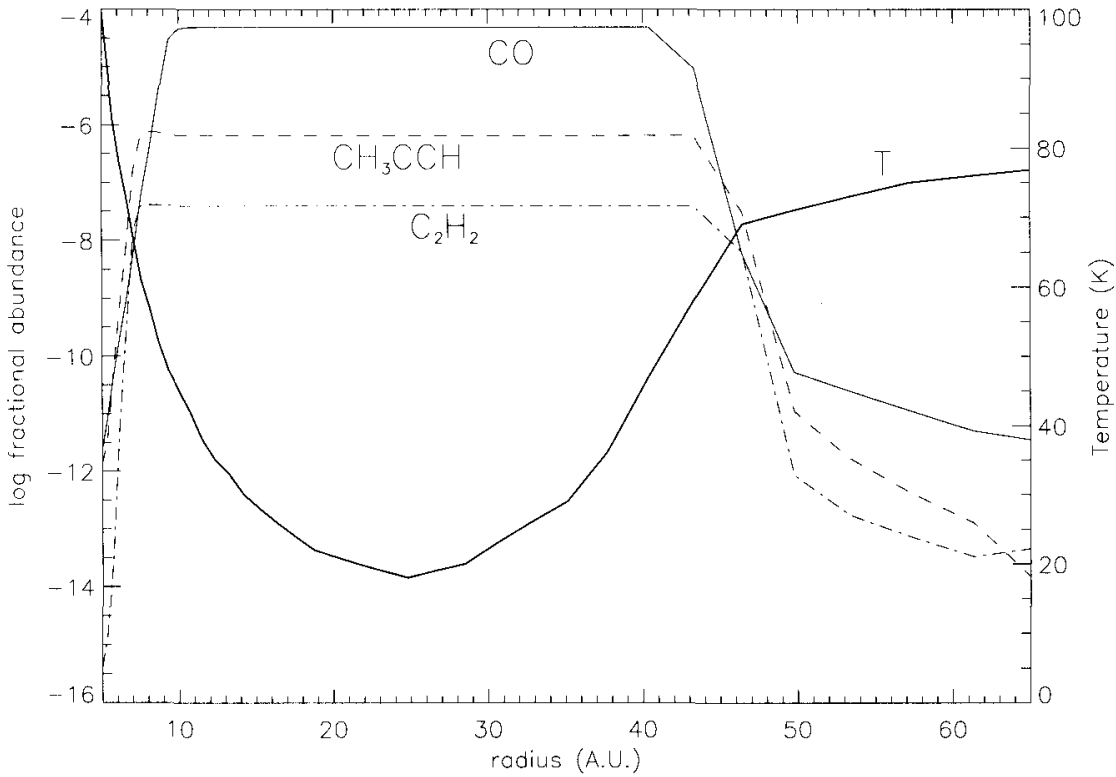

Figure 2. The mid-plane variation of the relative solid-phase abundances of $\mathrm{C}_{2} \mathrm{H}_{2}$ and $\mathrm{CH}_{3} \mathrm{CCH}$. The abundances are scaled to be the fractional abundance relative to gas-phase $\mathrm{H}_{2}$. Also shown is the temperature structure of the mid plane. 